\title{
Comparison of Staphylococcus aureus strains for ability to cause infective endocarditis and lethal sepsis in rabbits
}

\section{Adam R. Spaulding ${ }^{1}$, Erin A. Satterwhite ${ }^{2}$, Ying-Chi Lin ${ }^{3}$, Olivia N. Chuang-Smith ${ }^{4}$, Kristi L. Frank ${ }^{4}$, Joseph A. Merriman ${ }^{1}$, Matthew M. Schaefers ${ }^{3}$, Jeremy M. Yarwood ${ }^{5}$, Marnie L. Peterson ${ }^{3}$ and Patrick M. Schlievert ${ }^{1 *}$}

${ }^{1}$ Department of Microbiology, Carver College of Medicine, University of lowa, lowa City, IA, USA

23 Infection Prevention Division, 3M Company, St. Paul, Minneapolis, MN, USA

${ }^{3}$ Department of Experimental and Clinical Pharmacology, College of Pharmacy, University of Minnesota, Minneapolis, MN, USA

${ }^{4}$ Department of Microbiology, Medical School, University of Minnesota, Minneapolis, MN, USA

${ }^{5} 3 \mathrm{M}$ Corporate Research Laboratory, 3M Company, St. Paul, Minneapolis, MN, USA

Edited by:

Martin J. McGavin, University of Western Ontario, Canada

Reviewed by:

Francois Vandenesch, Immunity Infection Vaccination, France Vance Fowler, Duke University Medical Center, USA

\section{*Correspondence:}

Patrick M. Schlievert, Department of Microbiology, Carver College of Medicine, University of lowa, 51 Newton Road, 3-403 BSB, lowa City, IA 52242, USA. e-mail: patrick-schlievert@uiowa.edu
Staphylococcus aureus is a major cause of infective endocarditis (IE) and sepsis. Both methicillin-resistant (MRSA) and methicillin-sensitive (MSSA) strains cause these illnesses. Common S. aureus strains include pulsed-field gel electrophoresis (PFGE) types USA200, 300 , and 400 types where we hypothesize that secreted virulence factors contribute to both IE and sepsis. Rabbit cardiac physiology is considered similar to humans, and rabbits exhibit susceptibility to $S$. aureus superantigens (SAgs) and cytolysins. As such, rabbits are an excellent model for studying IE and sepsis, which over the course of four days develop IE vegetations and/or fatal septicemia. We examined the ability of MRSA and MSSA strains (4 USA200, 2 USA300, 2 USA400, and three additional common strains, FRI1169, Newman, and $\mathrm{COL})$ to cause vegetations and lethal sepsis in rabbits. USA200, TSST-1+ strains that produce only low amounts of $\alpha$-toxin, exhibited modest LD $D_{50}$ in sepsis $\left(1 \times 10^{8}-5 \times 10^{8}\right)$ colony-forming units (CFUs), and 3/4 caused significant IE. USA200 strain MNPE, which produces high-levels of $\alpha$-toxin, was both highly lethal (LD50 $5 \times 10^{6} \mathrm{CFUs}$ ) and effective in causing IE. In contrast, USA300 strains were highly effective in causing lethal sepsis ( $L D_{50} \mathrm{~S}$ $1 \times 10^{6}$ and $5 \times 10^{7}$ CFUs) but were minimally capable of causing IE. Strain Newman, which is phylogenetically related to USA300 strains, was not highly lethal (LD $D_{50}$ of $2 \times 10^{9}$ CFUs) and was effective in causing IE. USA400 strains were both highly lethal (LD50S of $1 \times 10^{7}$ and $5 \times 10^{7}$ CFUs) and highly effective causes of IE. The menstrual TSS isolate FRI1169, that is TSST-1+ ${ }^{+}$, produces high-levels of $\alpha$-toxin, but is not USA200, was both highly lethal and effective in causing IE. Additional studies showed that phenol soluble modulins (PSMs) produced by FRI1169 were important for sepsis but did not contribute to IE. Our studies show that these clonal groups of $S$. aureus differ in abilities to cause IE and lethal sepsis and suggest that secreted virulence factors, including SAgs and cytolysins, account for some of these differences.

Keywords: Staphylococcus aureus, exotoxins, infective endocarditis, sepsis

\section{INTRODUCTION}

Recently, the Centers for Disease Control and Prevention and collaborators published that Staphylococcus aureus is the most common cause of serious infectious diseases in the United States (Klevens et al., 2007). S. aureus is a common organism found in humans, with estimates of up to $40 \%$ of the population being colonized asymptomatically on mucosal and skin surfaces (Lowy, 1998; Schlievert et al., 2010). From these sites the organism causes many illnesses, ranging from benign soft tissue infections to life-threatening illnesses such as infective endocarditis (IE), sepsis, pneumonia, extreme pyrexia, and toxic shock syndrome (TSS) (Lowy, 1998; McCormick et al., 2001; Kravitz et al., 2005; Assimacopoulos et al., 2009). S. aureus is a serious pathogen both in hospital (Lowy, 1998) and community settings (Herold et al., 1998) with large numbers of severe infections emerging in the last decade.
In order to cause serious illnesses, S. aureus has many virulence factors that enable the microbe to interact with host tissues, defend itself from the immune system, and persist to cause organ dysfunction. Among the secreted virulence factors are multiple cytolysins and superantigens (SAgs) (Dinges et al., 2000; McCormick et al., 2001). The cytolysin $\alpha$-toxin has been known for many years to be required for $S$. aureus strains to cause dermonecrotic and inflammatory skin infections. Recently, $\alpha$-toxin has been shown to be important for causation of necrotizing pneumonia in mice (Bubeck Wardenburg et al., 2007; Bubeck Wardenburg and Schneewind, 2008). Other cytolysins include the hot:cold cytolysin $\beta$-toxin, which is a sphingomyelinase, biofilm ligase (Huseby et al., 2007, 2010), and participant in IE; $\delta$-toxin and other phenol soluble modulins (PSMs) (Otto, 2010), which lyse cells as either surfactants or by forming small pores; and the hetero-heptamer pore-forming toxins, including $\gamma$-toxin and 
Panton-Valentine leukocidin (Labandeira-Rey et al., 2007), may also contribute to serious illnesses.

Among the most studied secreted virulence factors are the SAgs, so named because of their unusual mechanism of dysregulating immune function (Marrack and Kappler, 1990; McCormick et al., 2001). SAgs include toxic shock syndrome toxin-1 (TSST-1), the emetic staphylococcal enterotoxins (SEs) serotypes A-E and I, and the non-emetic (or not tested) staphylococcal enterotoxin-like SAgs serotypes G, H, and J-X (McCormick et al., 2001). The biological activities of SAgs have been well described (Barsumian et al., 1978; Schlievert et al., 1981; Marrack and Kappler, 1990), and at least three (TSST$1, \mathrm{SEB}$, and SEC) cause the majority of cases of staphylococcal TSS (Schlievert et al., 2004). SAgs cause high fever (Schlievert et al., 1981; Schlievert, 1982), enhance host susceptibility to gramnegative lipopolysaccharide (Schlievert et al., 1981; Schlievert, 1982), and induce massive T-cell proliferation (Schlievert et al., 1981; Marrack and Kappler, 1990). SAgs stimulate T-cell proliferation by forming cross-bridges between the variable portions of the $\beta$-chains of the T-cell receptors (V $\beta$-TCRs) and invariant regions of the $\alpha$ - or $\beta$-chain of MHC II molecules on antigen presenting cells (Kotzin et al., 1993; Li et al., 1999; McCormick et al., 2001). SAg stimulation results in production of many cytokines, consequently leading to TSS. Recently, SAgs have been shown to induce proinflammatory responses in epithelial and endothelial cells, stimulating production primarily of chemokine responses, such as IL- 8 and MIP-3 $\alpha$, which may play important roles in the early stages of infection through outside-in signaling from mucosal surfaces (Brosnahan et al., 2009; Brosnahan and Schlievert, 2011).

IE is a life-threatening infection of the heart endothelium most often caused by gram-positive bacteria (Bashore et al., 2006), with S. aureus being one of the most common (Fowler et al., 2005). IE is characterized by the formation of "cauliflower-like" vegetations, comprised of host factors and microorganisms, on the damaged endothelium of heart valves. There are two major animal models for the study of IE, rats, and rabbits. Both models require that the aortic valves of animals be damaged, usually with hard plastic catheters threaded through the left carotid arteries and against the aortic valves for two or more hours. Often in the rat model, investigators leave the catheters in place for the duration of experimentation. It is suggested that in using this method, the ability of $S$. aureus to form biofilms on the catheters as well as aortic valves is being studied, complicating assessments of endocarditis; it is well recognized that foreign bodies greatly increase the ability of $S$. aureus to cause illness and make it difficult to determine the contribution of individual virulence factors. This model also suffers from the inability to assess the role of SAgs in IE since rodents are highly resistant to SAgs (Schlievert, 2009). We and others have extensively used rabbits where catheters are removed in the animals after aortic valves are damaged (Schlievert et al., 1998), and rabbits are highly susceptible to secreted virulence factors produced by $S$. aureus that have been tested thus far (Schlievert, 2009). In rabbits, vegetations can be seen as early as one day after intravenous microbial challenge, and vegetations can become large enough in four days to obstruct the aortas completely. Since $S$. aureus is administered to animals intravenously, we also gain important information on ability to cause lethal septicemia.

This study was undertaken to compare abilities of various $S$. aureus clonal lineages to cause IE and lethal sepsis in rabbits. We also examined the possible roles of selected cytolysins and SAgs in these infections.

\section{MATERIALS AND METHODS BACTERIA}

Well-characterized $S$. aureus isolates were tested for capacity to induce IE and lethal sepsis. Pulsed-field gel electrophoresis (PFGE) clonal group USA200 strains included menstrual TSS strains MN8 and CDC587 (Schlievert and Kelly, 1984), menstrual TSS community-associated methicillin-resistant (MRSA) S. aureus (CA-MRSA) MNWH, and post-influenza pneumonia TSS isolate MNPE (MacDonald et al., 1987). These strains produce TSST-1, and all except MNPE have a mutation in the $\alpha$-toxin gene that reduces the amount of the cytolysin produced by approximately 50-fold (see Table 1 for strains). We also studied menstrual TSS isolate FRI1169 and its naturally derived non-cytolytic variant termed JY3000. The variant emerged in biofilms from strain FRI1169, which is a TSST- $1^{+}, \alpha$-toxin high isolate (Yarwood et al., 2007); although from a patient with menstrual TSS, this strain does not belong to the USA200 clonal group based on dissimilarities in PFGE profiles. When cultured in the presence of serum and glucose, strain JY3000 became the dominant member of the FRI1169 population. Sequencing the agr locus in the organism yielded a single point mutation in agrA; however, this mutation did not explain the phenotype and gene expression patterns observed in the non-hemolytic variant. Microarray data confirmed that multiple virulence determinants were down-regulated, including agrACDB (9-fold), $\beta$ toxin (18-fold), RNAIII/ $\delta$-toxin (33-fold), $\gamma$-toxin (11-fold), and TSST-1 (5-fold). The following PFGE USA300 strains were used in our studies: CA-MRSA strain LAC, generously provided by Dr. F. R. DeLeo, NIH Rocky Mountain Laboratories, Hamilton, MT, and methicillin-sensitive (MSSA) strain MNLevy from a Minnesota case of extreme pyrexia complicating necrotizing pneumonia. USA400 strains included CA-MRSA MW2 and c99-529, both from the original description of necrotizing pneumonia in the upper Midwest (CDC, 1999). Finally, strain Newman, phylogenetically related to USA300 strains (Baba et al., 2008), and hospital-associated (HA)-MRSA strain COL were also evaluated. All strains used in these studies are maintained in the Schlievert laboratory as lyophilized stocks. For use in experimentation, the organisms were cultured on blood agar plates to ensure purity and then in Todd Hewitt broths (Difco Laboratories, Detroit, MI) at $37^{\circ} \mathrm{C}$ with 200 revolutions $/ \mathrm{min}$ shaking.

\section{RABBIT MODEL OF IE AND LETHAL SEPSIS}

IE and lethal sepsis were evaluated using New Zealand white rabbits with approval from IACUC (protocol 0908A71722) (Pragman and Schlievert, 2004; Pragman et al., 2004a). Rabbits were anesthetized with ketamine and xylazine. Once anesthetized, the aortic valves were mechanically damaged with hard plastic catheters inserted into the left carotid arteries. Two hours 
Table 1 | Lethality and infective endocarditis production by Staphylococcus aureus.

\begin{tabular}{|c|c|c|c|}
\hline S. aureus strain & $\begin{array}{l}\text { PFGE designation ( } \alpha \text {-Toxin } \\
\text { and Superantigen Profile) }\end{array}$ & $\begin{array}{l}\mathrm{LD}_{50} \text { after intravenous } \\
\text { injection after four days }\end{array}$ & Vegetation size \\
\hline Pulmonary TSS MSSA MNPE & USA200 ( $\alpha$-Toxin high, TSST $1^{+}$, SEC $\left.^{+}\right)$ & $5 \times 10^{6}$ & Up to $100 \mathrm{mg}$ \\
\hline Menstrual TSS MSSA MN8 & USA200 ( $\alpha$-Toxin low, TSST- $\left.1^{+}\right)$ & $5 \times 10^{8}$ & Up to $100 \mathrm{mg}$ \\
\hline Menstrual TSS CA-MRSA MNWH & USA200 ( $\alpha$-Toxinlow, TSST- $\left.1^{+}\right)$ & $2 \times 10^{8}$ & 0 \\
\hline Extreme Pyrexia and Necrotizing Pneumonia MSSA Levy & USA300 ( $\alpha$-Toxinhigh, SEI-X+) & $5 \times 10^{7}$ & Up to $20 \mathrm{mg}$ \\
\hline MSSA Newman & $\left(\alpha-\right.$ Toxin $^{\text {low }}$, SEA $\left.^{+}\right)$ & $2 \times 10^{9}$ & Up to $100 \mathrm{mg}$ \\
\hline Necrotizing Pneumonia CA-MRSA MW2 & USA400 $\left(\alpha-\right.$ Toxin $^{+}$, SEC4 $\left.{ }^{+}\right)$ & $5 \times 10^{7}$ & Up to $100 \mathrm{mg}$ \\
\hline Necrotizing Pneumonia CA-MRSA c99529 & USA400 $\left(\alpha-\right.$ Toxin $^{+}$, SEB $\left.^{+}\right)$ & $1 \times 10^{7}$ & Up to $100 \mathrm{mg}$ \\
\hline
\end{tabular}

Note: We investigated the ability of a number of representative clonal strains to cause IE and lethal sepsis in a rabbit model. Strains were grown overnight in Todd Hewitt broths at $37{ }^{\circ} \mathrm{C}$ shaking at $200 \mathrm{rpm}$. Dilutions were made (from $10^{5} / \mathrm{m} /$ to $4 \times 10^{9} / \mathrm{ml}$ ), and upon completion of surgery each rabbit was given a dose of $2 \mathrm{ml}$ of the appropriate strain. Numbers for the $L D_{50} s$ are given as the full $2 \mathrm{~m} /$ dose and were calculated to be the dose at which half of the rabbits died before the end of the 4-day trial.

later, catheters were removed, the rabbits were divided into groups, and the groups received varying doses of $S$. aureus strains washed one time and suspended in phosphate-buffered saline (PBS) intravenously in the marginal ear veins. The rabbits were allowed to awaken and were monitored daily for survival; rabbits were prematurely euthanized if they displayed symptoms $100 \%$ predictive of lethality (incapacity to right themselves and simultaneously failure to exhibit escape behavior) or euthanized (Beuthanasia D, Schering-Plough Animal Health Corp., Union, NJ) at the termination of experimentation after four days. Hearts were immediately removed to examine the aortas and aortic valves for the presence of vegetations, which were weighed.

\section{IMMUNIZATION STUDIES}

New Zealand white rabbits were immunized against a cocktail of PSMs $\alpha 1$, PSM $\alpha 4$, and $\delta$-toxin (PSM $\gamma$ ) and then challenged in the IE/sepsis model with strain FRI1169 as above. Peptides were synthesized and purified at the University of Minnesota Biomedical Genomics Center (>90\% purity by rHPLC). The lyophilized peptides were reconstituted in sterile distilled water. Rabbits received a series of three injections (days 0,14 , and 28) with the cocktail (120 $\mu \mathrm{g}$ of each per injection) diluted in PBS and then emulsified with incomplete Freund adjuvant (Difco Laboratories, Detroit, MI). Hyperimmunization (antibody titers > 2000) was verified by measuring the serum antibody titers to each antigen in all rabbits by ELISA. The immunized and non-immunized rabbits received $\sim 10^{7}$ CFUs of wildtype FRI1169 in the marginal ear veins.

\section{SUPERNATE PREPARATION}

Sterile supernates from 7 and $14 \mathrm{~h}$ cultures of strain FRI1169 and its non-cytolytic variant JY3000 grown in Todd Hewitt broths were collected by centrifugation followed by filtration (0.22 $\mu \mathrm{m}$; Millipore, Carrigtwohill, Co. Cork, Ireland). Protein was measured by Bio-Rad Protein Assay (Hercules, CA). To collect ethanol-insoluble exoproteins, supernates were treated with $80 \%$ final concentration $4^{\circ} \mathrm{C}$ ethanol and centrifuged $(1000 \times \mathrm{g}$, $15 \mathrm{~min})$. The ethanol-soluble fraction was lyophilized to collect exoproteins that did not precipitate. Ethanol (80\%) insoluble exoproteins were collected and dried. Both ethanol-soluble and insoluble fractions were reconstituted in ultrapure water to their original volumes.

\section{EXOPROTEIN CHARACTERIZATION}

Supernate proteins from FRI1169 and JY3000 were analyzed by sodium dodecyl-sulfate polyacrylamide gel electrophoresis (SDSPAGE) on 4-20\% gradient gels (Mini-PROTEAN TGX, Bio-Rad Laboratories, Inc.) and then either stained with Coomassie brilliant blue or silver (SilverXpress, Invitrogen, Carlsbad, CA). Unique protein bands were cut from the Coomassie-stained gels, analyzed using MALDI-MS, and compared against the database staphylococcus_NCBI_952306_CTM, to determine protein identity (University of Minnesota Center for Mass Spectrometry and Proteomics).

\section{TISSUE CULTURE EXPERIMENTS}

A549 human lung epithelial cells (ATCC, Manassas, VA) were grown in 96-well plates (Nalco, Naperville, IL) to $80 \%$ confluence in RPMI 1640 medium (Gibco, Invitrogen), supplemented with $10 \%$ fetal bovine serum (Sigma-Aldrich, St. Louis, MO) and an antibiotic cocktail $(25 \mu \mathrm{g} / \mathrm{ml}$ of penicillin, streptomycin, and fungizone; MP Biomedicals, Solon, $\mathrm{OH}$ ), and then changed to antibiotic-free medium overnight. The next day, strain FRI1169 or JY3000 supernates were diluted to $20 \mu \mathrm{g} / \mathrm{ml}$ protein in RPMI medium and used to replace the medium on the A549 cells. After $4 \mathrm{~h}$, interleukin-8 (IL-8) production was measured by ELISA (R\&D Systems, Minneapolis, MN), and cell viability was measured with the MTT-based reagent (Cell Titer 96 AQeous One, Promega, Madison WI). 


\section{$\alpha$-TOXIN WESTERN IMMUNOBLOTTING}

Ten microliters of filtered overnight culture supernates from strain FRI1169 or JY3000 were analyzed by 12\% SDS-PAGE, proteins transferred onto polyvinylidene fluoride (PVDF) membranes, and membranes immunoblotted with antiserum to $\alpha$-toxin (Sigma-Aldrich). Secondary, anti-rabbit IgG horseradish peroxidase-conjugated antibodies (Cell Signaling Technology, Danvers, MA), were employed for detection via chemoluminescence using SuperSignal West Dura Extended Duration substrate (Thermo Scientific).

\section{LD $_{50}$ AND STATISTICAL ANALYSES}

The $\mathrm{LD}_{50}$ of $S$. aureus strains following intravenous administration of washed bacteria, suspended in PBS, was determined by the method of Reed and Muench (Reed and Muench, 1938). Briefly, varying doses of $S$. aureus strains were administered to rabbits ( 3 per dose; $2 \mathrm{ml}$ per rabbit), with doses ranging from $10^{5} / \mathrm{ml}$ to $4 \times 10^{9} / \mathrm{ml}$. Deaths were recorded over a 4 -day time period. Tests for significance between means were carried out using Student's $t$-test or One-Way ANOVA with Dunnett post-test in Graph Prism (GraphPad Software, San Diego, CA). Significance in survival experiments was determined using Log-Rank Test (GraphPad Software).

\section{RESULTS}

\section{COMPARATIVE ABILITIES OF $\boldsymbol{S}$. aureus STRAINS TO CAUSE IE AND LETHAL SEPSIS}

We evaluated the ability of multiple $S$. aureus isolates to cause IE and lethal sepsis (Table 1). CDC clonal groups USA200, USA300, and USA400 strains, as well as other commonly used strains, were evaluated. All studies were performed in accordance with IACUC approval.

USA200 isolates are common strains found in IE patients (Xiong et al., 2006). USA200 strains, nearly all of which in our large collection produce $3-20 \mu \mathrm{g} / \mathrm{ml}$ of TSST- 1 in vitro in Todd Hewitt broths, were variably effective in causing vegetations of up to $100 \mathrm{mg}$, and there were differences in ability to cause lethal sepsis. The $\mathrm{LD}_{50}$ of the four USA200 strains, as determined by the method of Reed and Muench, ranged between $5 \times 10^{6}$ colonyforming units (CFU), for strain MNPE that has the wildtype $\alpha$-toxin gene, and $1-5 \times 10^{8} \mathrm{CFUs}$, for the strains that have mutations in the $\alpha$-toxin gene, thereby reducing $\alpha$-toxin production 50-fold (Lin et al., 2011). Interestingly, one vaginal isolate, CAMRSA MNWH, had an $\mathrm{LD}_{50}$ of $2 \times 10^{8}$ CFUs, comparable to the other strains with the $\alpha$-toxin gene mutation, but did not cause IE.

The prototypical USA300 strains differed from the USA200 strains in ability to cause illnesses. CA-MRSA LAC especially had a low $\mathrm{LD}_{50}$ of $1.2 \times 10^{6} \mathrm{CFUs}$, like USA200 strain MNPE, but did not cause vegetations, unlike MNPE. The lethal sepsis activity of LAC and MNPE correlated with high-level production of $\alpha$ toxin and production of SAgs, but the basis for lack of the ability of LAC to cause IE is unknown. MSSA USA300 strain MNLevy had a higher $\mathrm{LD}_{50}$ at $5 \times 10^{7}$ CFUs, but similarly caused only small vegetations. We have sequenced the genome of MNLevy and compared the sequence to the USA300 strain of Diep et al. (Diep et al., 2006); they are closely related, having a similar genome organization, except for the presence of the SCCmec DNA element in LAC. Strain Newman is not a USA300 strain but is phylogenetically related, and this organism appears to be unusually cardiotrophic in our studies, compared to other S. aureus tested. When injected intravenously, the organism caused extensive heart abscesses. Strain Newman caused IE, with large vegetations forming of up to $100 \mathrm{mg}$, but required more organisms to cause lethal sepsis ( $\left.\mathrm{LD}_{50} 2 \times 10^{9} \mathrm{CFUs}\right)$ than the two USA300 strains.

CA-MRSA USA400 strains MW2 and c99-529 was highly capable of causing both IE and lethal sepsis. MW2 had an $\mathrm{LD}_{50}$ of $5 \times 10^{7}$ CFUs and the ability to cause vegetations of up to $100 \mathrm{mg}$. C99-529 was similar, with an $\mathrm{LD}_{50}$ of $1 \times 10^{7}$ CFUs and similar ability to cause IE. MW2 is known to produce $\alpha$-toxin and the SAg SEC (Diep et al., 2008; Strandberg et al., 2010) and c99-529 produces $\alpha$-toxin, as determined by lysis of rabbit erythrocytes (data not shown), and SEB in high amounts (Strandberg et al., 2010) (50-100 $\mu \mathrm{g} / \mathrm{ml}$ in Todd Hewitt broths).

The HA-MRSA COL strain, which is not a USA400 strain but like many USA400 strains produces SEB in high amounts (Yarwood et al., 2002), was evaluated for its ability to cause IE and lethal sepsis; the strain was better at causing IE compared to other S. aureus strains, with vegetations reaching $200 \mathrm{mg}$ in agreement with a prior publication (Huseby et al., 2010), but doing so with a high $\mathrm{LD}_{50}$ of $2 \times 10^{9} \mathrm{CFU}$.

Collectively, our data suggest that USA200 and USA400 strains are generally better able to cause IE than USA300 strains. The presence of high-levels of cytolysins and SAgs correlates with increased ability to cause lethal sepsis.

\section{PSMS CONTRIBUTE TO LETHAL SEPSIS BUT NOT IE}

Studies have shown that cytolysins and SAgs contribute to IE (Cheung et al., 1994; Pragman et al., 2004a; Huseby et al., 2010). However, studies have not evaluated the role of PSMs in IE. Through studies initiated with S. aureus FRI1169 and a noncytolytic, natural variant JY3000, we evaluated the role. Our studies showed that wildtype FRI1169 is both highly lethal $\left(\mathrm{LD}_{50}\right.$ $5 \times 10^{6} \mathrm{CFUs}$ ) and capable of causing IE (Table 1).

Based on our past experience with USA200 strain MNPE, in which lethal sepsis appeared to correlate with high $\alpha$-toxin production, we hypothesized that FRI1169 lethality would be due to its wildtype $\alpha$-toxin production. We thus initiated studies to compare the cytotoxicity of wildtype FRI1169 and the natural biofilm mutant JY3000 organism. A549 cells were exposed to early $(7 \mathrm{~h})$ and late stationary phase $(14 \mathrm{~h})$ culture supernates from both organisms for $4 \mathrm{~h}$ to compare cytotoxicity and pro-inflammatory responses. Supernates from two time points of growth were used to ensure that exoproteins expressed at different points were included. Epithelial cells were selected because they serve as a primary barrier to $S$. aureus infection on mucosal surfaces. Application of the $7 \mathrm{~h}$ supernates from wildtype FRI1169 resulted in approximately 50\% reduction in cell viability, and the $14 \mathrm{~h}$ supernates resulted in over $90 \%$ reduction in cell viability (Figure 1A). In contrast, neither of the variant JY3000 supernates caused viability changes relative to media controls. The pro-inflammatory response, measured by IL- 8 production to attract polymorphonuclear leukocytes, 
A

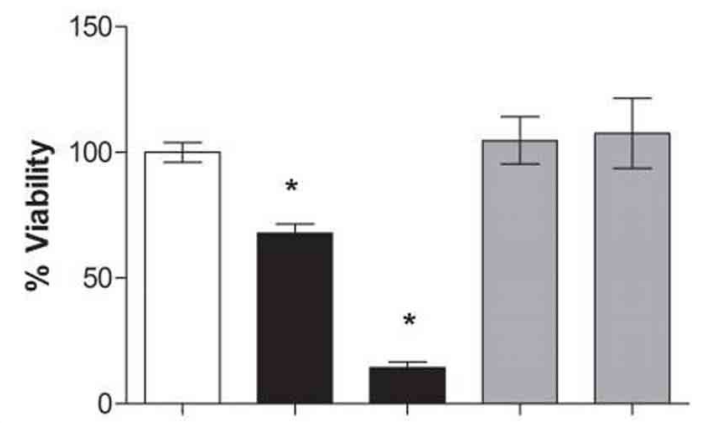

B

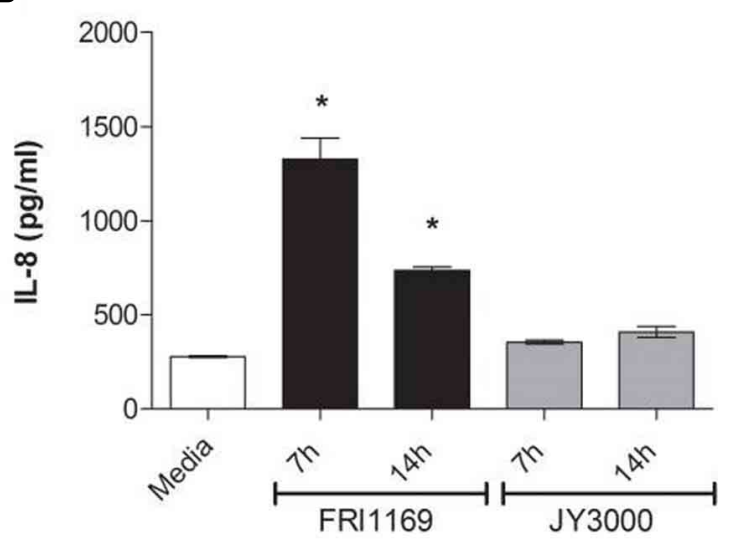

FIGURE 1 | In vitro comparison of wildtype FRI1169 and variant JY3000 cytotoxicity. A549 cell cytotoxicity (A) and IL-8 response (B) after $4 \mathrm{~h}$ exposure to $S$. aureus broth culture supernates of FRI1169 (wildtype cytolytic) and JY3000 (biofilm-derived variant, non-cytolytic) grown for 7 or $14 \mathrm{~h} .{ }^{*}$ Indicates $p<0.05$ compared to medium control (RPMI medium alone) by One-Way ANOVA, data plotted as mean \pm standard deviation.

also confirmed difference between wildtype FRI1169 and variant JY3000 (Figure 1B). Supernates from JY3000 at both time points did not alter IL- 8 production relative to the medium-only control. However, supernates from wildtype FRI1169 caused significant increases in IL-8 production. We hypothesized that the major differences between FRI1169 and JY3000 cytolytic and proinflammatory activities depended on differential production of $\alpha$-toxin, but as shown below the major differences were related to differential production of PSMs, including $\delta$-toxin.

Supernates from 7 and $14 \mathrm{~h}$ broth cultures of FRI1169 and JY3000 were diluted to $20 \mu \mathrm{g} / \mathrm{ml}$ protein and subjected to SDSPAGE. A unique band was observed for wildtype FRI1169 that was not present in the variant JY3000 fluids. This was a thick band containing low molecular weight species present in abundance in the $14 \mathrm{~h}$ FRI1169 supernates, and to a lesser extent in the 7 h FRI1169 supernates (Figure 2A). Aside from the difference in pattern, in three independent experiments we observed less total exoprotein in variant JY3000 supernates than in wildtype FRI1169 fluids ( $p=0.005$, Figure 2B).

Secreted virulence factors such as $\alpha$-toxin and TSST- 1 are known to be insoluble in $80 \%$ ethanol while smaller molecules are soluble in $80 \%$ ethanol, including cytolytic peptides known

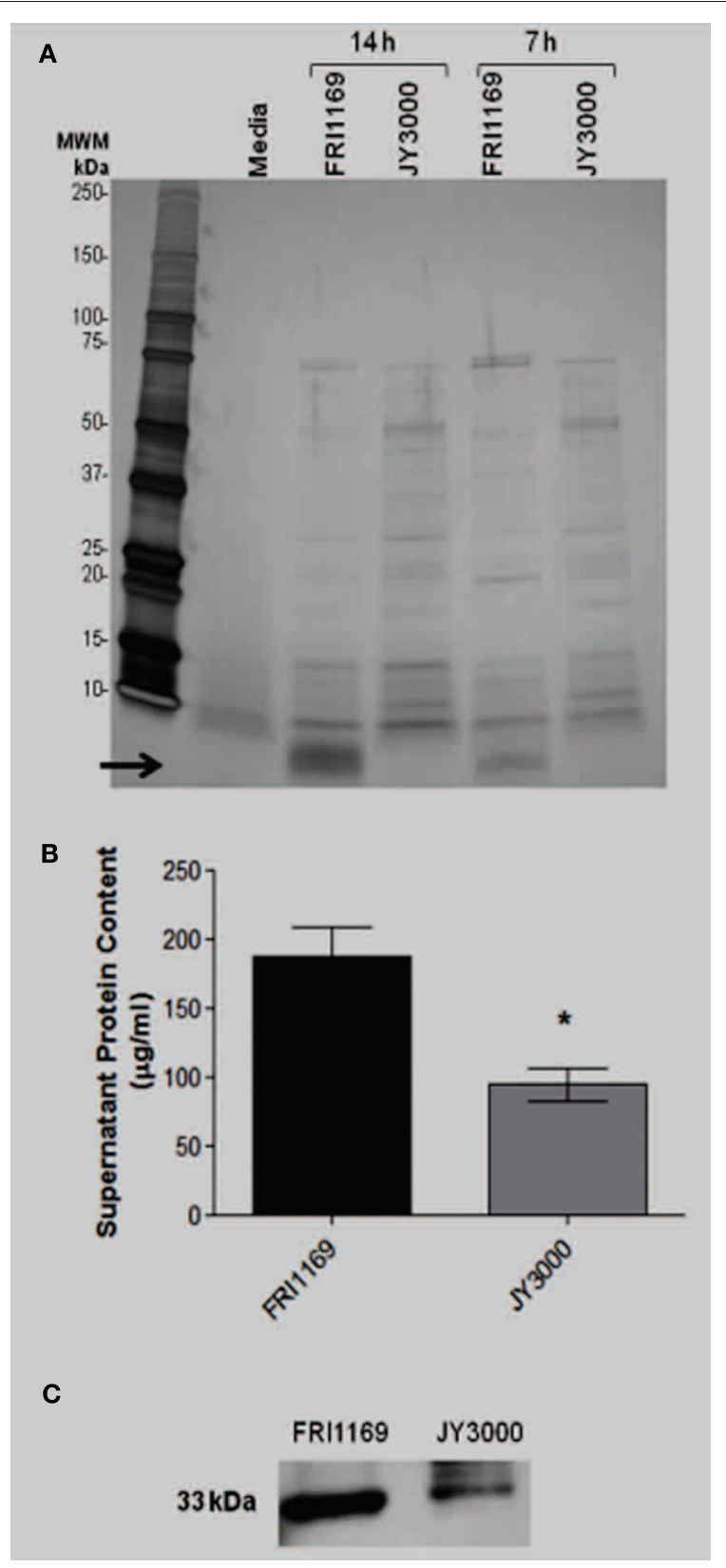

FIGURE 2 | Characterization of exoprotein production by wildtype FRI1169 and variant JY3000. (A) 14 and $7 \mathrm{~h}$ broth culture supernates (20 $\mu \mathrm{g}$ of protein loaded per lane) were separated by SDS-PAGE and silver-stained. Media denotes lane electrophoresed with Todd Hewitt media alone. Arrow denotes small molecular weight $(<10 \mathrm{kDa})$ band only present in FRI1169 cultures. (B) Total protein content measured in three separate overnight broth culture supernates from JY3000 and FRI1169 via Bio Rad reagent. ( $P=0.005$ by Student's $t$-test). (C) Western immunoblot results showing differences in $\alpha$-toxin amounts produced by wildtype FRI1169 and JY3000.

as PSMs. We demonstrated that wildtype FRI1169 and variant JY3000 produced detectable $\alpha$-toxin by Western immunoblot, with FRI1169 producing more than JY3000. Late stationary phase supernates of wildtype FRI1169 were prepared and subjected to $80 \%$ ethanol treatment. Both the ethanol-insoluble 
and ethanol-soluble fractions were reconstituted to their original volumes and compared using SDS-PAGE. The low molecular weight band observed in Figure 2 was present exclusively in the ethanol-soluble fraction (Figure 3A). A549 cells were exposed to the reconstituted ethanol-soluble and ethanolinsoluble fractions, as well as the whole supernates. After $4 \mathrm{~h}$, A549 cells exposed to the wildtype FRI1169 ethanol-soluble fractions and whole supernates showed reduced cell viability (Figure 3B). Surprisingly, no reduction in viability was detected for the re-solubilized wildtype FRI1169 ethanol precipitate, where $\alpha$-toxin and TSST- 1 were present. None of the JY3000 supernates demonstrated cytotoxicity (Figure 3B).

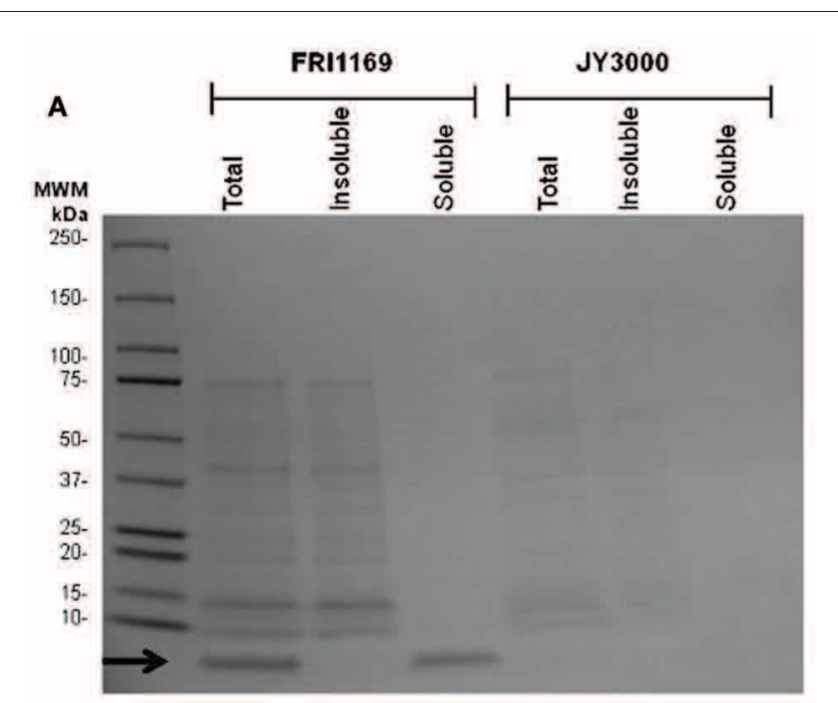

B

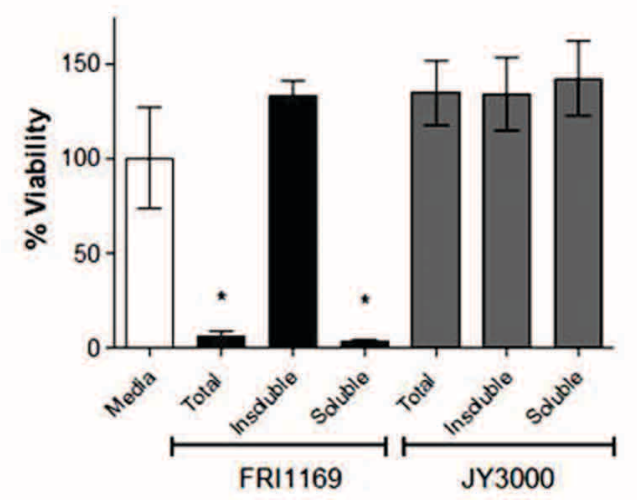

FIGURE 3 | Ethanol (80\%) separation of exoprotein and determination of cytotoxicity. (A) 14 and $7 \mathrm{~h}$ broth culture supernatant fluids ( $20 \mu \mathrm{g}$ of protein was loaded per lane) were treated with $80 \%$ (final concentration) ethanol and total, ethanol-insoluble, and ethanol-soluble fractions were separated by SDS-PAGE and Coomassie brilliant blue-stained. Arrow denotes small molecular weight $(<10 \mathrm{kDa})$ band only present in total supernatant fluid or ethanol soluble fraction of FRI1169 culture (B) A549 cells were exposed to total, ethanol insoluble, and ethanol soluble fractions of FRI1169 and JY3000. Cytotoxicity was measured and plotted relative to media only (untreated) control. (*Indicates $P<0.05$ relative to media control).
Because of the association between the presence of the low molecular weight species in wildtype FRI1169 and cytotoxicity for A549 cells, we analyzed the band via MALDI-MS analysis to identify the protein(s). Unique peptides were mapped to three, (potentially four) virulence elements: PSM $\alpha-1, \operatorname{PSM} \alpha-2$ (redundant sequence with $\alpha-1$ ), PSM $\alpha-4$, and $\delta$-toxin (PSM $\gamma$ ).

The rabbit IE/sepsis model was selected to understand preliminarily whether the in vitro cytotoxicity observations were predictive of in vivo virulence. Rabbits were injected with either wildtype FRI1169 or variant JY3000 at a concentration of $\sim 10^{8}$ CFUs/injection. After developing symptoms of serious infections, rabbits administered wildtype FRI1169 died within $24 \mathrm{~h}$ $(n=5)$ as a result of sepsis. Conversely, rabbits administered an equivalent dose of variant JY3000 survived with mild symptoms over the same time period. This experiment was terminated on day 2, as no FRI1169-inoculated rabbits survived long enough to develop significant aortic vegetations.

Since there are 3-4 PSMs that could account for cytotoxicity of strain FRI1169, and because one of these is $\delta$-toxin in which its mRNA is RNA III, a global regulator of exotoxin production, it was straightforward to do immunization studies to assess their role in virulence, rather than attempt to make knockouts in the 3-4 PSMs. We thus immunized rabbits against PSM $\alpha-1, \operatorname{PSM} \alpha-4$, and $\delta$-hemolysin (PSM $\gamma$ ), verified that the animals were hyperimmunized by ELISA, and then challenged them and non-immune controls with $\sim 2.5 \times 10^{7}$ CFUs wildtype FRI1169. By $36 \mathrm{~h}$ post-inoculation, 5/5 of the non-immunized rabbits succumbed, while 4/4 immunized rabbits remained alive. Over the next $36 \mathrm{~h}$, the immunized rabbits also died, indicating immunization delayed death, but ultimately did not block lethality ( $p$ value $=0.008$, Figure 4 ). The rabbits presumably ultimately succumbed to TSS due to wildtype production of TSST-1. Vegetations on the aortic valves were recovered from all of the non-immunized rabbits (ranging from 1.0 to $9.0 \mathrm{mg}$ per valve) and from 3/4 immunized rabbits (ranging from 0.5

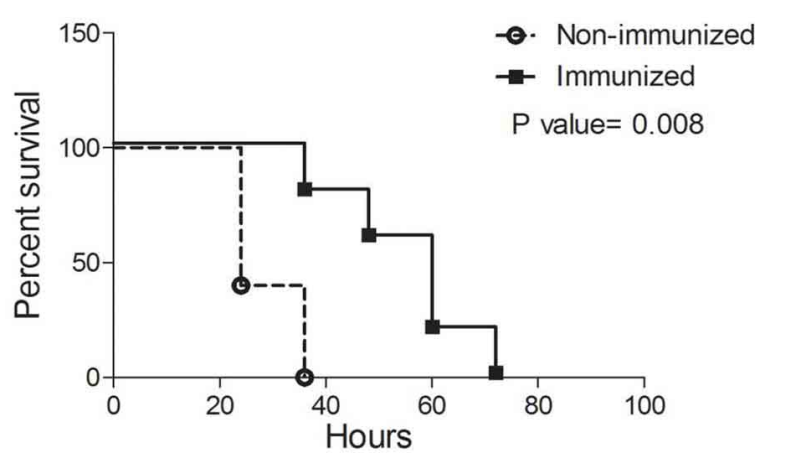

FIGURE 4 | Effect of immunization against PSMs on lethal sepsis/infectious endocarditis. Rabbits ( $n=4$ or 5 per group) were either immunized against a cocktail of PSM $\alpha-1$, PSM $\alpha-4$, and PSM $\gamma / \delta$-toxin (squares) or left unimmunized (circles). Aortic valves were then mechanically damaged, and rabbits were challenged with $\sim 10^{7}$ CFUs of wildtype FRI1169 in the marginal ear veins. Rabbits were monitored for up to four days and were prematurely euthanized if displayed signs predictive of lethal illness. $P$-value determined by log rank test. 
to $11.7 \mathrm{mg}$ per valve). There was no significant difference in the number or weights of vegetations between the immunized and non-immunized rabbits.

\section{DISCUSSION}

The present studies have shown that there are differences among clonal groups of $S$. aureus with respect to causing IE and fatal sepsis. In general USA200 strains cause IE and have modest lethal activity in rabbits. In contrast, USA300 strains are only weakly able to cause IE, but the strains are highly lethal to rabbits. USA400 strains interestingly are both effective in causing IE and fatal sepsis. Our studies also show that PSMs, at least as produced by strain FRI1169, are important in causation of fatal sepsis in rabbits but do not contribute in major ways to IE.

We and others suggest that important cytolysins and SAgs contribute to the ability of strains to cause these and other illnesses. For example, USA200 isolates are the primary causes of TSS, accounting for nearly all menstrual TSS cases and $50 \%$ of non-menstrual TSS cases, including post-influenza cases (Bergdoll et al., 1981; Schlievert et al., 1981; MacDonald et al., 1987). These isolates and the additional menstrual TSS strain FRI1169 all produce TSST-1 as their dominant SAg. These strains lack the recently described SAg SEl-X which has been associated with necrotizing pneumonia caused by USA300 strains (Wilson et al., 2011). The majority of vaginal USA200 isolates, have a stop codon in the $\alpha$-toxin gene $(h l a)$, preventing them from making wildtype amounts of $\alpha$-toxin. Interestingly, these isolates have developed a mechanism by which they read through the stop codon and produce small amounts of $\alpha$-toxin (Lin et al., 2011). MNPE and FRI1169 produce high-amounts of $\alpha$-toxin as tested in vitro (up to $50 \mu \mathrm{g} / \mathrm{ml}$ ). USA300 and USA400 strains, mainly CA-MRSA, are especially capable of causing skin and soft tissue abscesses and necrotizing pneumonia. Staphylococcal $\alpha$-toxin is required for causation of skin infections (Kobayashi et al., 2011), and both $\alpha$-toxin and SAgs, including a newly described SAg SEl-X, are required for fatal necrotizing pneumonia (CDC, 1999; Fey et al., 2003; Bubeck Wardenburg et al., 2007; Strandberg et al., 2010; Wilson et al., 2011).

The role of exotoxins in IE and lethal sepsis is only partially defined. In 1994, Cheung et al. showed that sar ${ }^{-} /$agr ${ }^{-}$mutants were reduced in their abilities to colonize heart endothelium and cause IE, indicating that the regulation of production of exotoxins by these two component regulatory systems is critical for S. aureus to cause IE in a rabbit model (Cheung and Projan, 1994). Similarly, Xiong et al. showed that $\alpha$-toxin regulation by sae is critical in vivo in the rabbit model for IE in that mutants, that had reduced sae activity and concurrent reduced hla production, were reduced in their abilities to cause IE compared to a wildtype isolate (Xiong et al., 2006). The SAg TSST-1 has been shown to be critical for IE in a rabbit model. Pragman et al. showed that TSST- $1^{-}$strains of $S$. aureus have much lower abilities to cause endocarditis than isogenic TSST- $1^{+}$strains (Pragman et al., 2004b). The TSST- ${ }^{+}$strains had much larger vegetations and on average $1 \times 10^{6}$ CFUs more per vegetations than TSST- ${ }^{-}$ strains (Pragman et al., 2004b). It is not known why the TSST- $1^{+}$ CA-MRSA USA200 MNWH did not cause vegetations, but clearly the pro-IE role of TSST-1 can be modified by other factors in this strain.

In recent studies, Huseby et al. showed the pivotal role of $\beta$-toxin in IE (Huseby et al., 2010). Strain COL, known to produce $\beta$-toxin, was better able to cause vegetations than the COL strain knocked-out for $\beta$-toxin through bacteriophage integration into the $\beta$-toxin structural gene. While many $S$. aureus strains causing human illness do not produce $\beta$-toxin, many USA200 strains produce the toxin. USA200 strains are generally highly effective in causing endocarditis, and this may in part be due to the biofilm ligase activity of $\beta$-toxin (Huseby et al., 2010).

The data from our studies indicate that for $S$. aureus strain FRI1169, PSMs are important in determination of lethal sepsis, but are not critical for production of IE. These data are in agreement with prior studies of CA-MRSA USA300 strains that suggest PSMs contribute significantly to serious illnesses (Otto, 2010).

Finally, in agreement with the above studies, Seidl et al. recently showed that the ability of a $S$. aureus strain to induce endothelial damage in vitro was positively correlated to its ability to cause disease in a rabbit model of IE (Seidl et al., 2011). Taken together these data suggest that SAgs, cytolysins, and their regulatory mechanisms make for a highly virulent combination and are required for the progression of IE.

Recently, the Interscience Conference on Antimicrobial Agents and Chemotherapy published a historical account of their first 50 years. In that publication, it was noted that major symposia have been held each year to assess progress in management of IE. Additionally, large numbers of manuscript are published yearly studying IE. These symposia and papers indicate a clear need to continue research into understanding the fundamentals of IE caused by $S$. aureus to better treat patients and reduce the number of cases each year. We stress the importance of evaluating the role of the secreted virulence factors in these diseases, as many published studies have shown that both SAgs and cytolysins play definitive roles. It is only through a thorough understanding of their contributions in sensitive animal models that we will be able to find novel strategies to manage the illness. Our studies also demonstrate that different clonal groups, and even within clonal groups, variation in disease potential exists, making it difficult to make global statements about causative factors for groups of strains.

\section{ACKNOWLEDGMENTS}

We thank the Center for Mass Spectrometry and Proteomics at the University of Minnesota for the MALDI-MS analysis, the Biomedical Genomic Center at the University of Minnesota for synthesizing the phenol soluble modulins. This research was funded by USPHS research grants AI-74283 (PMS), AI57153 (PMS) from the NIH-supported Great Lakes Regional Center for Excellence in Biodefense and Emerging Diseases where PMS is a member, and AI-73366 (MLP) from the National Institute of Allergy and Infectious Diseases, and 3M Non-tenured unrestricted faculty research award (MLP). 


\section{REFERENCES}

Assimacopoulos, A. P., Strandberg, K. L., Rotschafer, J. H., and Schlievert, P. M. (2009). Extreme pyrexia and rapid death due to Staphylococcus aureus infection: analysis of 2 cases. Clin. Infect. Dis. 48, 612-614.

Baba, T., Bae, T., Schneewind, O., Takeuchi, F., and Hiramatsu, K. (2008). Genome sequence of Staphylococcus aureus strain Newman and comparative analysis of staphylococcal genomes: polymorphism and evolution of two major pathogenicity islands. J. Bacteriol. 190, 300-310.

Barsumian, E. L., Schlievert, P. M., and Watson, D. W. (1978). Nonspecific and specific immunological mitogenicity by group A streptococcal pyrogenic exotoxins. Infect. Immun. 22, 681-688.

Bashore, T. M., Cabell, C., and Fowler, V. Jr. (2006). Update on infective endocarditis. Curr. Probl. Cardiol. $31,274-352$.

Bergdoll, M. S., Crass, B. A., Reiser, R. F., Robbins, R. N., and Davis, J. P. (1981). A new staphylococcal enterotoxin, enterotoxin $\mathrm{F}$, associated with toxic- shock-syndrome Staphylococcus aureus isolates. Lancet 1, 1017-1021.

Brosnahan, A. J., Mantz, M. J., Squier, C. A., Peterson, M. L., and Schlievert, P. M. (2009). Cytolysins augment superantigen penetration of stratified mucosa. J. Immunol. 182, 2364-2373.

Brosnahan, A. J., and Schlievert, P. M. (2011). Gram positive bacterial superantigen outside-in signaling causes toxic shock syndrome. FEBS J. 278, 4649-4667.

Bubeck Wardenburg, J., Bae, T., Otto, M., Deleo, F. R., and Schneewind, O. (2007). Poring over pores: alphahemolysin and Panton-Valentine leukocidin in Staphylococcus aureus pneumonia. Nat. Med. 13, 1405-1406.

Bubeck Wardenburg, J., and Schneewind, O. (2008). Vaccine protection against Staphylococcus aureus pneumonia. J. Exp. Med. 205, 287-294.

CDC, (1999). From the centers for disease control and prevention. Four pediatric deaths from communityacquired methicillin-resistant Staphylococcus aureus - Minnesota and North Dakota, 1997-1999. JAMA 282, 1123-1125.

Cheung, A. L., Eberhardt, K. J., Chung, E., Yeaman, M. R., Sullam, P. M., Ramos, M., and Bayer, A. S. (1994). Diminished virulence of a sar-/agrmutant of Staphylococcus aureus in the rabbit model of endocarditis. J. Clin. Invest. 94, 1815-1822.

Cheung, A. L., and Projan, S. J. (1994) Cloning and sequencing of sarA of Staphylococcus aureus, a gene required for the expression of agr. J. Bacteriol. 176, 4168-4172.

Diep, B. A., Gill, S. R., Chang, R. F., Phan, T. H., Chen, J. H. Davidson, M. G., Lin, F., Lin, J., Carleton, H. A., Mongodin, E. F., Sensabaugh, G. F., and PerdreauRemington, F. (2006). Complete genome sequence of USA300, an epidemic clone of communityacquired meticillin-resistant Staphylococcus aureus. Lancet 367, 731-739.

Diep, B. A., Palazzolo-Ballance, A. M., Tattevin, P., Basuino, L., Braughton, K. R., Whitney, A. R., Chen, L., Kreiswirth, B. N., Otto, M., Deleo, F. R., and Chambers, H. F. (2008). Contribution of Panton-Valentine leukocidin in community-associated methicillinresistant Staphylococcus aureus pathogenesis. PLoS One 3, e3198. doi: 10.1371/journal.pone.0003198

Dinges, M. M., Orwin, P. M., and Schlievert, P. M. (2000). Exotoxins of Staphylococcus aureus. Clin. Microbiol. Rev. 13, 16-34.

Fey, P. D., Said-Salim, B., Rupp, M. E., Hinrichs, S. H., Boxrud, D. J., Davis, C. C., Kreiswirth, B. N., and Schlievert, P. M. (2003). Comparative molecular analysis of community- or hospitalacquired methicillin-resistant Staphylococcus aureus. Antimicrob. Agents Chemother. 47, 196-203.

Fowler, V. G. Jr., Miro, J. M., Hoen, B., Cabell, C. H., Abrutyn, E., Rubinstein, E., Corey, G. R., Spelman, D., Bradley, S. F., Barsic, B., Pappas, P. A., Anstrom, K. J., Wray, D., Fortes, C. Q., Anguera, I., Athan, E., Jones, P., van der Meer, J. T., Elliott, T. S., Levine, D. P., and Bayer, A. S. (2005). Staphylococcus aureus endocarditis: a consequence of medical progress. JAMA 293, 3012-3021.

Herold, B. C., Immergluck, L. C., Maranan, M. C., Lauderdale, D. S., Gaskin, R. E., Boyle-Vavra, S., Leitch, C. D., and Daum, R. S. (1998). Community-acquired methicillin-resistant Staphylococcus aureus in children with no identified predisposing risk. JAMA 279, 593-598.

Huseby, M., Shi, K., Brown, C. K., Digre, J., Mengistu, F., Seo, K. S., Bohach, G. A., Schlievert, P. M., Ohlendorf, D. H., and Earhart, C. A. (2007). Structure and biological activities of beta toxin from
Staphylococcus aureus. J. Bacteriol. 189, 8719-8726.

Huseby, M. J., Kruse, A. C., Digre, J., Kohler, P. L., Vocke, J. A., Mann, E. E., Bayles, K. W., Bohach, G. A., Schlievert, P. M., Ohlendorf, D. H., and Earhart, C. A. (2010). Beta toxin catalyzes formation of nucleoprotein matrix in staphylococcal biofilms. Proc. Natl. Acad. Sci. U.S.A. 107, 14407-14412.

Klevens, R. M., Morrison, M. A., Nadle, J., Petit, S., Gershman, K., Ray, S., Harrison, L. H., Lynfield, R. Dumyati, G., Townes, J. M., Craig, A. S., Zell, E. R., Fosheim, G. E., Mcdougal, L. K., Carey, R. B., and Fridkin, S. K. (2007). Invasive methicillin-resistant Staphylococcus aureus infections in the United States. JAMA 298, 1763-1771.

Kobayashi, S. D., Malachowa, N., Whitney, A. R., Braughton, K. R. Gardner, D. J., Long, D., Bubeck Wardenburg, J., Schneewind, O., Otto, M., and Deleo, F. R. (2011). Comparative analysis of USA300 virulence determinants in a rabbit model of skin and soft tissue infection. J. Infect. Dis. 204 937-941.

Kotzin, B. L., Leung, D. Y., Kappler, J., and Marrack, P. (1993). Superantigens and their potential role in human disease. $A d v$ Immunol. 54, 99-166.

Kravitz, G., Dries, D. J., Peterson, M. L., and Schlievert, P. M (2005). Purpura fulminans due to Staphylococcus aureus. Clin. Infect. Dis. 40, 941-947.

Labandeira-Rey, M., Couzon, F., Boisset, S., Brown, E. L., Bes, M., Benito, Y., Barbu, E. M. Vazquez, V., Hook, M., Etienne, J. Vandenesch, F., and Bowden, M. G. (2007). Staphylococcus aureus Panton-Valentine leukocidin causes necrotizing pneumonia. Science 315, 1130-1133.

Li, H., Llera, A., Malchiodi, E. L., and Mariuzza, R. A. (1999). The structural basis of $\mathrm{T}$ cell activation by superantigens. Annu. Rev. Immunol. 17, 435-466.

Lin, Y. C., Anderson, M. J., Kohler, P. L., Strandberg, K. L., Olson, M E., Horswill, A. R., Schlievert, P. M., and Peterson, M. L. (2011) Proinflammatory exoprotein characterization of toxic shock syndrome Staphylococcus aureus. Biochemistry 50, 7157-7167.

Lowy, F. D. (1998). Staphylococcus aureus infections. N. Engl. J. Med. $339,520-532$

MacDonald, K. L., Osterholm, M. T., Hedberg, C. W., Schrock, C. G. Peterson, G. F., Jentzen, J. M.,
Leonard, S. A., and Schlievert, P. M. (1987). Toxic shock syndrome. A newly recognized complication of influenza and influenzalike illness. JAMA 257, 1053-1058.

Marrack, P., and Kappler, J. (1990). The staphylococcal enterotoxins and their relatives. Science 248, 705-711.

McCormick, J. K., Yarwood, J. M., and Schlievert, P. M. (2001). Toxic shock syndrome and bacterial superantigens: an update. Annu. Rev. Microbiol. 55, 77-104.

Otto, M. (2010). Basis of virulence in community-associated methicillinresistant Staphylococcus aureus. Annu. Rev. Microbiol. 64, 143-162.

Pragman, A. A., and Schlievert, P. M. (2004). Virulence regulation in Staphylococcus aureus: the need for in vivo analysis of virulence factor regulation. FEMS Immunol. Med. Microbiol. 42, 147-154.

Pragman, A. A., Yarwood, J. M. Tripp, T. J., and Schlievert, P. M. (2004a). Characterization of virulence factor regulation by SrrAB, a two-component system in Staphylococcus aureus. J. Bacteriol. 186, 2430-2438.

Pragman, A. A., Yarwood, J. M., Tripp, T. J., and Schlievert, P. M. (2004b). Characterization of virulence factor regulation by SrrAB, a two-component system in Staphylococcus aureus. J. Bacteriol. 186, 2430-2438.

Reed, L. J., and Muench, H. (1938). A simple method of estimating fifty percent end points. Am. J. Hyg. 27, 493-497.

Schlievert, P. M. (1982). Enhancement of host susceptibility to lethal endotoxin shock by staphylococcal pyrogenic exotoxin type C. Infect. Immun. 36, 123-128.

Schlievert, P. M. (2009). Cytolysins, superantigens, and pneumonia due to community-associated methicillin-resistant Staphylococcus aureus. J. Infect. Dis. 200, 676-678.

Schlievert, P. M., Gahr, P. J., Assimacopoulos, A. P., Dinges, M. M., Stoehr, J. A., Harmala, J. W., Hirt, H., and Dunny, G. M. (1998). Aggregation and binding substances enhance pathogenicity in rabbit models of Enterococcus faecalis endocarditis. Infect. Immun. 66, 218-223.

Schlievert, P. M., and Kelly, J. A. (1984). Clindamycin-induced suppression of toxic-shock syndrome-associated exotoxin production. J. Infect. Dis. $149,471$.

Schlievert, P. M., Shands, K. N., Dan, B. B., Schmid, G. P., and Nishimura, R. D. (1981). Identification and characterization of an exotoxin 
from Staphylococcus aureus associated with toxic-shock syndrome. J. Infect. Dis. 143, 509-516.

Schlievert, P. M., Strandberg, K. L., Lin, Y. C., Peterson, M. L., and Leung, D. Y. (2010). Secreted virulence factor comparison between methicillinresistant and methicillin-sensitive Staphylococcus aureus, and its relevance to atopic dermatitis. J. Allergy Clin. Immunol. 125, 39-49.

Schlievert, P. M., Tripp, T. J., and Peterson, M. L. (2004). Reemergence of Staphylococcal Toxic Shock Syndrome in Minneapolis-St. Paul, Minnesota, during the 2000-2003 Surveillance Period. J. Clin. Microbiol. 42, 2875-2876.

Seidl, K., Bayer, A. S., Mckinnell, J. A., Ellison, S., Filler, S. G., and Xiong, Y. Q. (2011). In vitro endothelial cell damage is positively correlated with enhanced virulence and poor vancomycin responsiveness in experimental endocarditis due to methicillinresistant Staphylococcus aureus. Cell. Microbiol. 13, 1530-1541.

Strandberg, K. L., Rotschafer, J. H., Vetter, S. M., Buonpane, R. A., Kranz, D. M., and Schlievert, P. M. (2010). Staphylococcal superantigens cause lethal pulmonary disease in rabbits. J. Infect. Dis. 202, 1690-1697.

Wilson, G. J., Seo, K. S., Cartwright, R. A., Connelley, T., Chuang-Smith, O. N., Merriman, J. A., Guinane, C. M., Park, J. Y., Bohach, G. A., Schlievert, P. M., Morrison, W. I., and Fitzgerald, J. R. (2011). A novel core genome-encoded superantigen contributes to lethality of community-associated MRSA necrotizing pneumonia. PLoS Pathog. 7, e1002271. doi: 10.1371/journal.ppat.1002271

Xiong, Y. Q., Willard, J., Yeaman, M. R., Cheung, A. L., and Bayer, A. S. (2006). Regulation of Staphylococcus aureus alpha-toxin gene (hla) expression by agr, sarA, and sae in vitro and in experimental infective endocarditis. J. Infect. Dis. 194 1267-1275.

Yarwood, J. M., McCormick, J. K. Paustian, M. L., Orwin, P. M., Kapur, V., and Schlievert, P. M. (2002). Characterization and expression analysis of Staphylococcus aureus pathogenicity island 3. Implications for the evolution of staphylococcal pathogenicity islands. J. Biol. Chem. 277, 13138-13147.

Yarwood, J. M., Paquette, K. M., Tikh, I. B., Volper, E. M., and Greenberg, E. P. (2007). Generation of virulence factor variants in Staphylococcus aureus biofilms. J. Bacteriol. 189 7961-7967.

Conflict of Interest Statement: Erin A. Satterwhite and Jeremy M. Yarwood are employees of 3M Company, St. Paul, MN, USA. Marnie L. Peterson has research grants with $3 \mathrm{M}$ for unrelated studies. None of the other authors have a conflict of interest to declare.

Received: 06 January 2012; accepted: 07 February 2012; published online: 21 February 2012.

Citation: Spaulding AR, Satterwhite EA, Lin Y-C, Chuang-Smith ON, Frank KL, Merriman JA, Schaefers MM, Yarwood $J M$, Peterson $M L$ and Schlievert PM (2012) Comparison of Staphylococcus aureus strains for ability to cause infective endocarditis and lethal sepsis in rabbits. Front. Cell. Inf. Microbio. 2:18. doi: 10.3389/fcimb.2012.00018

Copyright (C) 2012 Spaulding, Satterwhite, Lin, Chuang-Smith, Frank, Merriman, Schaefers, Yarwood, Peterson and Schlievert. This is an open-access article distributed under the terms of the Creative Commons Attribution Non Commercial License, which permits non-commercial use, distribution, and reproduction in other forums, provided the original authors and source are credited. 